\title{
Efficacy of a combination of $10 \%$ imidacloprid and $1 \%$ moxidectin against Caparinia tripilis in African pygmy hedgehog (Atelerix albiventris)
}

\author{
Kyu-Rim Kim ${ }^{1 \dagger}$, Kyu-Sung Ahn ${ }^{2 \dagger}$, Dae-Sung $\mathrm{Oh}^{2}$ and Sung-Shik Shin ${ }^{1 *}$
}

\begin{abstract}
Background: The efficacy and safety of a combination formulation of $10 \%$ imidacloprid $+1.0 \%$ moxidectin spot-on (Advocate ${ }^{\circledR}$ for Cats, Bayer Animal Health $\mathrm{GmbH}$, Leverkusen, Germany) was tested in 40 African pygmy hedgehogs (Atelerix albiventris) naturally infested with Caparinia tripilis.

Methods: The optimal dosage level of the combination for hedgehogs was determined by assigning 20 hedgehogs into three treatment groups $(0.1,0.4$ and $1.6 \mathrm{ml} / \mathrm{Kg} \mathrm{b.w.)}$, and one untreated control group of 5 hedgehogs each. Twenty naturally infested hedgehogs were then randomly assigned to either treatment or control group with 10 animals each, and the number of live mites was counted from 13 body regions on day 0, 3, 9, 16, and 30 after single treatment at the dosage level of $0.1 \mathrm{ml} / \mathrm{Kg}$.

Results: Before the chemotherapy, the highest density of mite was observed in external ear canals followed by the dorsal and the lowest in the ventral regions of the body surface. The dosage level of $0.1 \mathrm{ml} / \mathrm{Kg}$, which corresponded to the recommended dosage level for cats, containing $10 \mathrm{mg}$ imidacloprid and $1 \mathrm{mg}$ moxidectin was also the optimal dosage level for hedgehogs. No hedgehogs in the treatment group showed live mites from day 3 post treatment. Side effects such as ataxia, depression, nausea, and weight fluctuation were not observed during the whole period of study.
\end{abstract}

Conclusions: This report suggests that a combination formulation of $0.1 \mathrm{ml} / \mathrm{Kg}$ of $10 \%$ imidacloprid $+1 \%$ moxidectin spot-on for cats is also useful for the control of Caparinia tripilis infestation in hedgehogs.

Keywords: African pygmy hedgehog, Atelerix albiventris, Caparinia tripilis, moxidectin, imidacloprid

\section{Background}

Caparinia tripilis (Michael, 1889), first reported in Erinaceus europaeus from England [1], is one of the most important causes of skin disease in hedgehogs [2]. The ears of infested animals become scaly and the skin of the affected parts thickens. The mite causes annoyance to their hosts, which shows pruritic symptoms as they often sit on their haunches and attempt to scratch infested areas with their hind claws. In advanced cases, dry crusts form on the surface of the skin, which thickens, becomes folded, and may even crack. Bleeding can occur when the lesions become worse, spines may fall out, and

\footnotetext{
* Correspondence: sungshik@jnu.ac.kr

${ }^{+}$Equal contributors

'Department of Parasitology, College of Veterinary Medicine, Chonnam

National University, Gwangju 500-757, Korea

Full list of author information is available at the end of the article
}

affected hedgehogs eventually become so incapacitated that they cannot roll up properly [3]. The life cycle of $C$. tripilis includes egg, larva, protonymph, deutonymph (none of which shows sexual dimorphism) and either an adult male or an adult female [3]. Observations suggest that the complete life cycle encompasses about three weeks [3]. By means of genital suckers, an adult male and a pubescent female (deutonymph) form an attachment pair, which is more or less permanent up until the time of emergence of the adult female from the deutonymphal exuviae. Severe infestation may be found in young animals as early as the age of 4 months and heavily infested animals may die [3]. Infested hedgehogs, therefore, must be isolated and treated.

Treatments in hedgehogs have included organophosphate bath, ivermectin injection, and amitraz spray $[4,5]$.

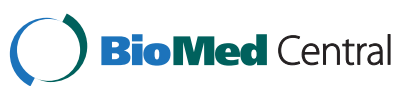


After the treatment with organophosphate bath (Paramite $^{\mathrm{TM}}$, Vet-Kem Co., Dallas, Texas), skin lesions were improved and the condition had not recurred [4]. However, organophosphate insecticide toxicity is the leading cause of major morbidity and death in the insecticide class. The clinical syndrome of organophosphate toxicity varies widely, ranging from the classic cholinergic syndrome to flaccid paralysis and even intractable seizures [6]. While ivermectin (Eqvalan ${ }^{\circledR}$, Merck \& Co., Rahway, New Jersey) administered intramuscularly at 21-day intervals reduced clinical disease caused by Caparinia infestation in $A$. albiventris, it failed to eliminate the mites. On the other hand, two treatments with a $0.03 \%$ rinse of amitraz (Mitaban ${ }^{\circledR}$, Upjohn Co., Kalamazoo, Michigan) 7 days apart cleared the infestation [5]. An application of $1 \%$ permethrin has also been shown to be effective [7].

Moxidectin, a macrocyclic lactone disaccharide, is a potent, broad-spectrum endectocide with activity against a wide range of nematodes, insects, and mites. First used commercially in Argentina as an injectable formulation for cattle in 1989, it has been widely used as a broadspectrum antiparasitic remedy for a variety of mammalian species including food-producing and companion animals [8]. It has been reported that moxidectin is 100 times more lipophilic than ivermectin and that the concentration of moxidectin in fat tissue was 90 -fold higher than that detected in plasma 28 days following treatment in cattle $[9,10]$. The high liphophilic nature of moxidectin may be particularly important as a potent miticide against those parasitic mites that live on the surface of animal skin with sebaceous glands. Recent experimental studies confirmed the efficacy of topical imidacloprid + moxidectin against otoacariosis caused by Otodectes cynotes in dogs [11] and cats [12]. The imidacloprid + moxidectin formulation were also highly efficacious against the KS1 Ctenocephalides felis, flea strain infesting cats [13]. With the ease of a spot-on medication, the formulation could also be used for parasitic control in exotic pet animals, and studies on ear mite infection caused by $O$. cynotes in ferrets resulted in successful elimination of the parasite [14]. However, the efficacy and side effects against mange infestation in hedgehogs have not been studied yet. The objective of the present investigation is to evaluate the efficacy and safety of imidacloprid $10 \%+$ moxidectin $1 \%$ spot-on against naturally infested Caparinia tripilis in hedgehogs.

\section{Methods}

\section{Study animals}

In November 2010, forty African pygmy hedgehogs (Atelerix albiventris, 13 male and 27 female, mean \pm SD body weight, $305.6 \pm 111.2$ g) naturally infested with Caparinia tripilis were obtained from a local pet shop in
Gwangju, Korea. A brief description of the hedgehogs' sex, body weight, and coloring was recorded. None of the hedgehogs had been treated with ectoparasiticides in the last 6 months before the experiment. The hedgehogs were kept in cages individually during the experiment. All animals were housed in the same room at $25.0 \pm 2.0^{\circ}$ $\mathrm{C}$ and $80 \%$ relative humidity, and were given regular commercial hedgehog food throughout the experimental period. The Institutional Animal Care and Use Committee at Chonnam National University approved the protocols used in this study, and the animals were cared for in accordance with the Guidelines for Animal Experiments.

For parasitological identification, skin scraping samples from severely affected animals were collected and preserved in $70 \%$ methanol. Mites were mounted on slides using Downs' PVA solution for several days until the internal organs became transparent [15], and were identified by the morphology of adult males according to a key provided by Laurence [16].

\section{Drug}

For the treatment against the mite infestation, a formulation product of $10 \%$ imidacloprid $+1 \%$ moxidectin spot-on for cats (Advocate ${ }^{\circledR}$ for Cats, Bayer Animal Health GmbH, Leverkusen, Germany) was used.

\section{Study designs \\ Experiment 1. Determination of optimal dosage level for hedgehogs}

Fifteen hedgehogs were randomly assigned to the treatment group and five to the untreated control group by a random treatment allocation plan generated with a Microsoft ${ }^{\circledR}$ Excel macro (Microsoft Corporation, Redmond, WA, USA). The treatment group was divided into 3 subgroups of 5 hedgehogs each for 3 different dosage levels of the test drug. Treatment groups were administered once with different dosage levels of the test drug (0.1, 0.4 or $1.6 \mathrm{ml} / \mathrm{Kg}$ b.w.) on day 0 to the skin of the mid-dorsal region of each hedgehog using a pipette (Gilson Pipetman, p200, France) after the number of mites was counted. The control group received the same aliquot of $0.1 \%$ benzyl alcohol. The dosage level of $0.1 \mathrm{ml} /$ Kg contained $10 \mathrm{mg}$ imidacloprid and $1 \mathrm{mg}$ moxidectin.

The main criterion for the drug efficacy was the absence of viable mites from the host animal at any developmental stage, including larva, nymph, and adult. The distinction of gender was not attempted because the nymph and adult stages were particularly difficult to differentiate on a fresh preparation unless the internal nontransparent organs and materials were cleared by mounting liquids such as PVA solution, which takes several days [15]. Mites were considered as live if they were motile and retained their body conformation. Enumeration of mites by conventional skin scraping and light 
microscopic examination was not used in this study because dead mites killed by the drug administration could erroneously be counted as live mites during light microscopic examination after $\mathrm{KOH}$ dissolution. Likewise, the ovicidal efficacy of the test drug was not evaluated because the viability of eggs could not be easily determined either by otoscopic or microscopic examination. Although no records for the genus Caparinia are available, eggs of the genera Chorioptes and Otodectes which are closely related to Caparinia in the family Psoroptidae, require an average of 4 days (range 72-107 hours) to hatch at $35^{\circ} \mathrm{C}$ and $80 \%$ relative humidity $[17,18]$. Therefore, successful control of eggs can be expected if larvicidal efficacy of the test drug is ensured to last at least for one week post treatment.

Before the administration of the drug on day 0 , the number of live motile mites were counted from a total of 13 skin regions of each animal (6 dorsal [dorsal neck, left and right dorsal thoracic, left and right lumbar, and sacral regions], 5 ventral [left and right pectoral, left and right abdominal and inguinal regions] and external ears of both sides) using an otoscope (Piccolight ${ }^{\circledR}$, KaWe, Berlin, Germany) for 30 seconds each. The number of mites on approximately $0.5 \mathrm{~cm}^{2}$ of body surface was counted for each region. On day 3 , the number of live mites found in all twenty hedgehogs was counted by the same method as in Day 0. Every hedgehog was weighed with a scale to observe weight change at each examination.

\section{Experiment 2. Efficacy evaluation}

Ten naturally infected hedgehogs were randomly assigned to the treatment group and ten naturally infected hedgehogs to the untreated control group by a random treatment allocation plan generated with a Microsoft ${ }^{\circledR}$ Excel macro (Microsoft Corporation, Redmond, WA, USA). Before the administration of the drug, the number of live mites found in all twenty hedgehogs was counted by the same methods as described in Experiment 1. Advocate ${ }^{\circledR}$ for Cats (Bayer Animal Health $\mathrm{GmbH}$, Leverkusen, Germany) at the dosage level of 0.1 $\mathrm{ml} / \mathrm{Kg}$ was administered to the skin of the mid-dorsal part of hedgehogs in the treatment group as a single treatment on day 0 . All animals were examined 5 times, on day $0,3,9,16$, and 30 during the experiment. The number of live mites found in 13 body regions was counted by the same method as described in Experiment 1. After all 13 body regions were examined by an otoscope, the viability of mites was double checked by collecting skin scraping samples from the posterior dorsal neck region of each hedgehog into a disposable polystyrene dish (IWAKI 150x20mm, Asahi Glass Co., Ltd., Japan) and examined under a stereoscope (Stemi 2000$\mathrm{C}$, Zeiss) for live mites. All animals were weighed with a scale on each examination. The efficacy of the drug in each treatment group for Day 3 and after was calculated as follows:

The percentage of efficacy $(\%)=\frac{y-x}{y} \times 100$

$\mathrm{x}=$ number of hedgehogs observed with live mites

$\mathrm{y}=$ total number of hedgehogs in group

\section{Statistical analysis}

A Kruskal-Wallis test was used to evaluate differences in the population density between body regions. Differences in body weight measured over time were evaluated through repeated-measure ANOVA models. The signification level was set at $\mathrm{p} \leq 0.05$. Statistical analysis was performed using IBM SPSS Statistics software version 19.0 (IBM Corporation, Armonk, New York, U.S.).

\section{Results}

Figure 1 shows a stereoscope view of the affected skin of a naturally-infested hedgehog with numerous mites at various developmental stages. The mantle of severely populated regions of the skin was covered with scale and crust that were often carpeted with either live eggs or empty egg shells (Figure 2). Morphologically, pedicels of adult mites were short and unjointed. Tarsal caruncles were bell-shaped on all legs of males while they were absent on legs III and IV of females. Three long setae on the third pair of legs in both sexes were present. Adult males had posterior end of the abdomen with trilobate projection on each side and each lobe with a long seta (Figure 3). Based on these morphological characteristics, the mite was identified as Caparinia tripilis.

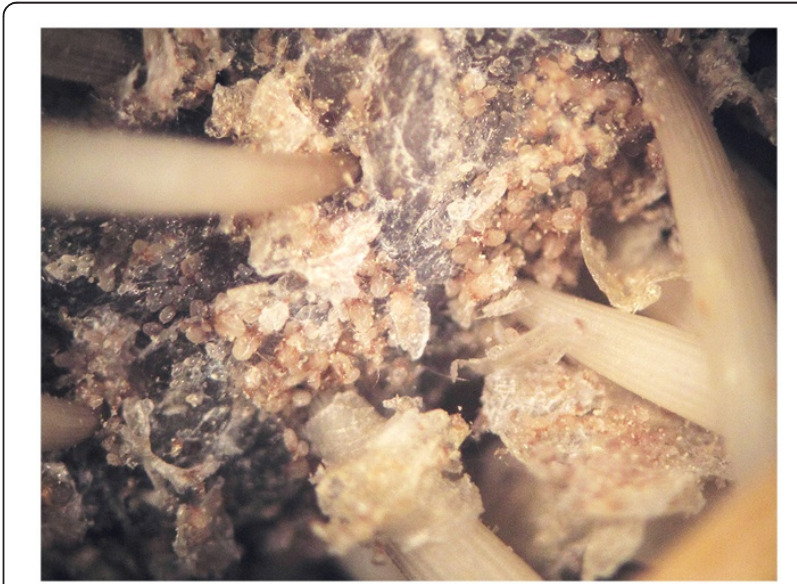

Figure 1 Heavily-infested skin of a hedgehog (Atelerix albiventris) with Caparinia tripilis. Numerous mites at various developmental stages including eggs are shown. Photo image taken from a dissecting microscope (Zeiss Stemi 2000-C). 

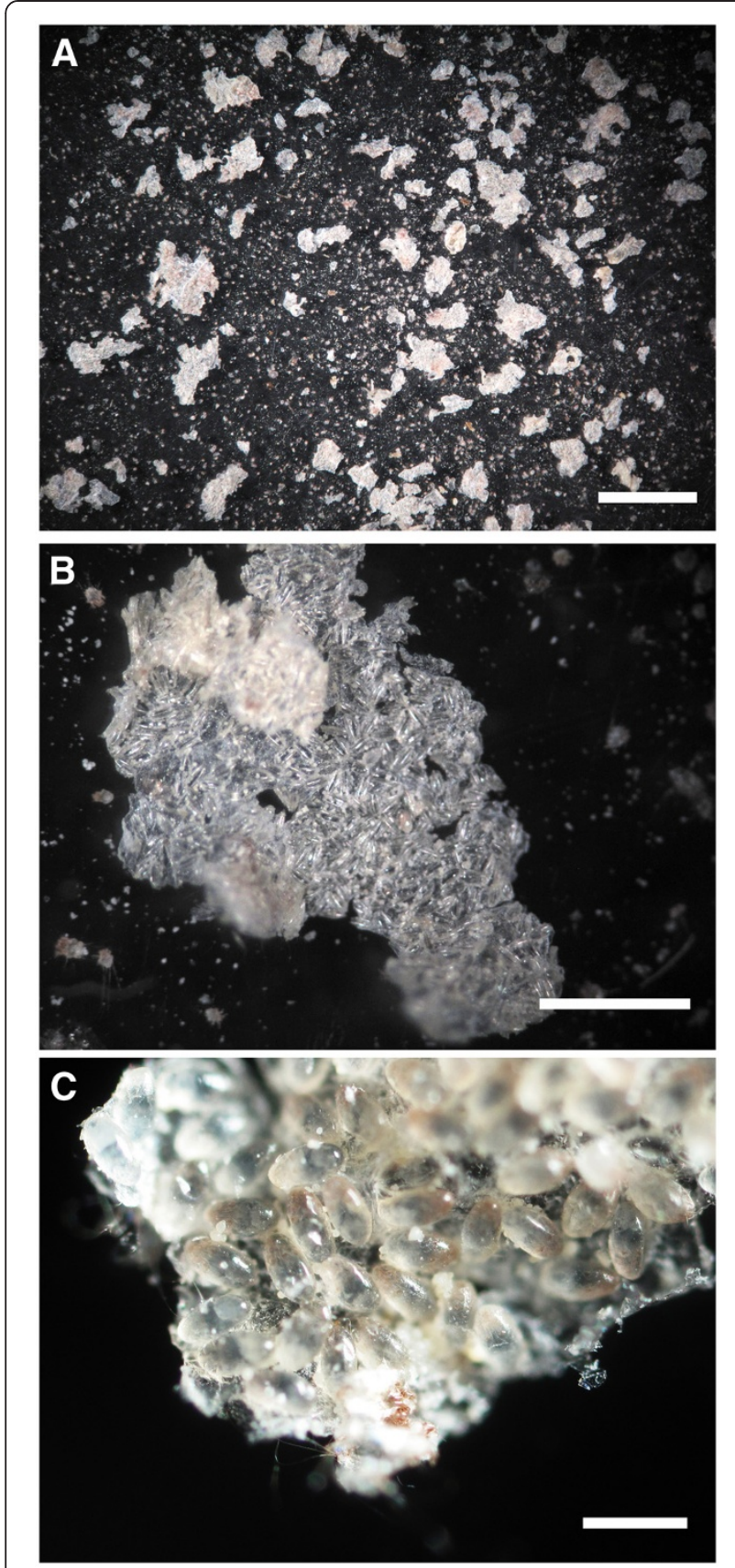

Figure 2 Flakes of scale collected from a hedgehog heavily infested with Caparinia tripilis. A, Low magnification of scales collected from an infested hedgehog. Scales were stored at room temperature for 4 weeks. Tiny dots of larvae hatched from eggs are shown. Bar $=5 \mathrm{~mm}$. B, Photograph of an enlarged scale that is covered with mostly empty egg shells. Bar $=1 \mathrm{~mm}$. C, Photomicrograph of an enlarged scale of a heavily-infested hedgehog showing numerous eggs. $\operatorname{Bar}=200 \mu \mathrm{m}$.

The distribution pattern of C. tripilis on the 13 body regions of 40 hedgehogs before chemotherapy is shown in Table 1. Among the total of 2,495 mites counted from the 13 body regions with mean number of $4.8 \pm 5.9$ mites (mean \pm S.D.), the highest density of mite was observed in the left external ear canal $(8.7 \pm 8.5)$, and the lowest in the inguinal region $(0.9 \pm 1.8)$. In general, the greatest concentration was recognized in ears $(8.5 \pm$ $8.7)$, followed by dorsal $(6.3 \pm 5.5)$, and the lowest in the ventral regions $(1.5 \pm 2.4)$. The difference in the number of mites between ears, dorsal, and ventral regions was significant with the Kruskal-Wallis test $(\mathrm{p}<0.001)$.

\section{Experiment 1. Determination of proper dosage level}

Results for the optimal dosage level of Advocate ${ }^{\circledR}$ for Cats against $C$. tripilis infestation in hedgehogs are shown in Table 2. Most mites in the treatment group administered with a dosage level of $0.1 \mathrm{ml} / \mathrm{Kg}$ were found dead on day 3. Only one live mite was found in one of five animals at the dorsal neck region. Other treatment groups, which were administered at the dosage level of 0.4 and $1.6 \mathrm{ml} / \mathrm{Kg}$, respectively, showed no live mites at any body region examined. On day 3 in the control group, on the other hand, the mean number of mites was maintained above 3.7 with the highest density found in the left ear and the lowest in the inguinal region. There were no side effects recognized even at the dosage level of $1.6 \mathrm{ml} / \mathrm{Kg}$, which was equivalent to sixteen times the recommended dosage level for cats. A dosage level of 0.1 $\mathrm{ml} / \mathrm{Kg}$ was therefore selected as the optimal dosage for hedgehogs for Experiment 2.

\section{Experiment 2. Efficacy evaluation of drug}

A long-term miticidal efficacy of a formulation of $10 \%$ imidacloprid $+1 \%$ moxidectin against $C$. tripilis is shown in Table 3. While the average number of mites on the 13 body regions in the untreated control group maintained above 4.7 during the experimental period, hedgehogs in the treatment group showed no live mites from day 3 post treatment until the end of the experiment. The efficacy of $10 \%$ imidacloprid $+1 \%$ moxidectin on C. tripilis in hedgehogs was, therefore, $100 \%$ from day 3 to day 30 . The efficacy of the drug was double confirmed by observing the viability of mites in skin scraping samples from the posterior dorsal neck region of each hedgehog under a stereoscope from which no live mites were observed from animals in the treatment group (Figure 4, data not shown).

The average weights of the control and treatment groups were $254 \pm 88.2 \mathrm{~g}$ and $290 \pm 164.1 \mathrm{~g}$ on day 0 , respectively. On day 30 , the average weight of the control group remained unchanged $(254.0 \pm 53.8 \mathrm{~g})$ while that of the treatment group increased slightly $(297.0 \pm$ $137.1 \mathrm{~g})$. The body weight change from day 0 to 30 was not statistically significant in both control $(\mathrm{p}=0.830)$ and treatment $(p=0.198)$ groups. Side effects such as ataxia, 


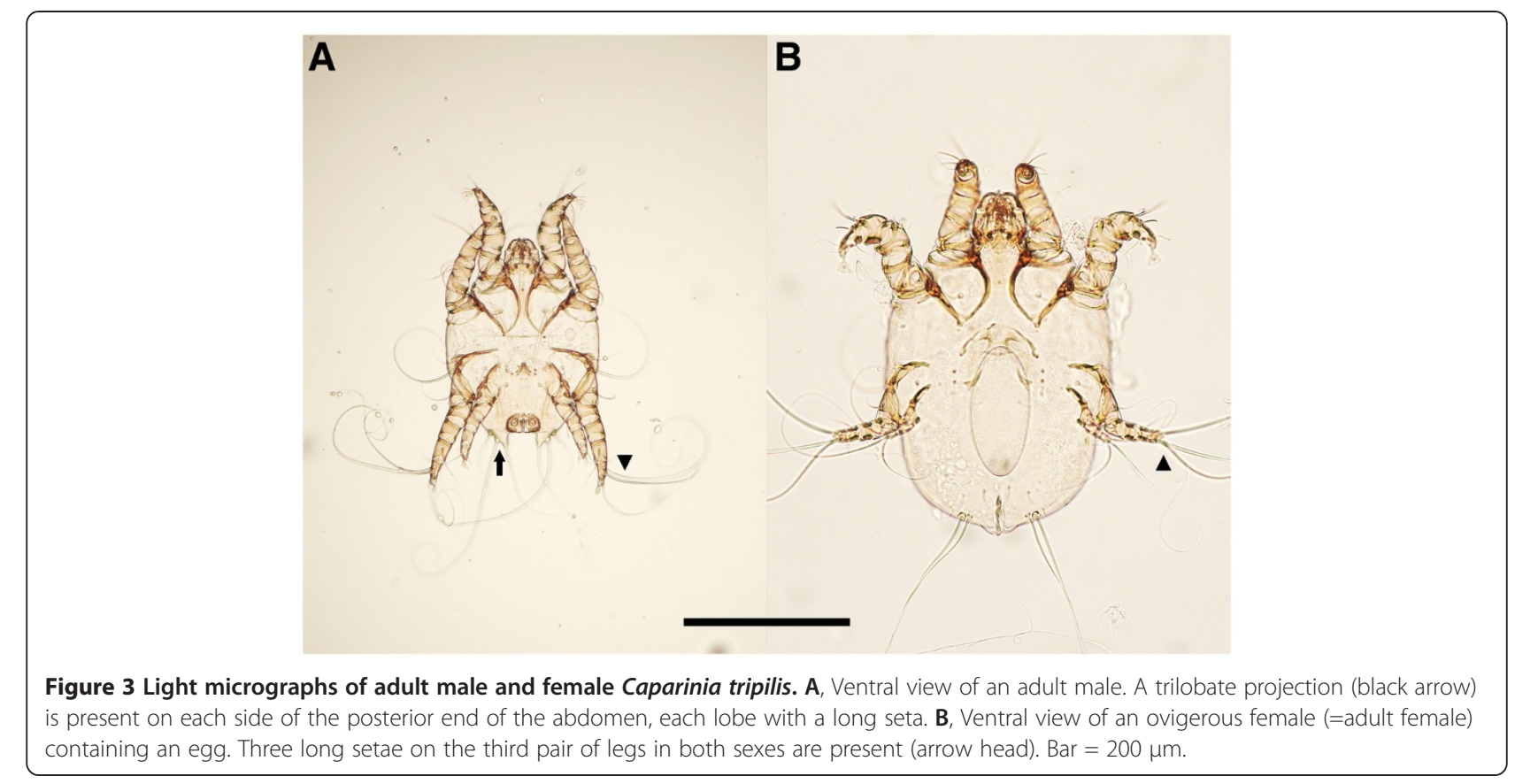

depression, or nausea were not observed during the whole period of study in both control and treatment groups.

Table 1 Distribution of Caparinia tripilis on 13 body regions of $\mathbf{4 0}$ African pygmy hedgehogs (Atelerix albiventris)

\begin{tabular}{|c|c|c|c|c|}
\hline \multicolumn{2}{|c|}{ Body Regions } & \multicolumn{3}{|c|}{ Number of Caparinia tripilis counted } \\
\hline & & Total & Mean & S.D. \\
\hline \multirow[t]{3}{*}{ Ears } & $\underline{\text { Left }}$ & 340 & 8.7 & 8.5 \\
\hline & Right & 323 & 8.3 & 8.9 \\
\hline & Subtotal & 663 & 8.5 & 8.7 \\
\hline \multirow[t]{6}{*}{ Ventral } & Left pectoral & 86 & 2.2 & 2.6 \\
\hline & Right pectoral & 73 & 1.8 & 2.8 \\
\hline & Left abdominal & 47 & 1.2 & 1.3 \\
\hline & Right abdominal & 66 & 1.7 & 2.9 \\
\hline & Inguinal & 37 & 0.9 & 1.8 \\
\hline & Subtotal & 309 & 1.5 & 2.4 \\
\hline \multirow[t]{7}{*}{ Dorsal } & Dorsal neck & 327 & 8.2 & 6.5 \\
\hline & Left dorsal thoracic & 303 & 7.6 & 6.8 \\
\hline & Right dorsal thoracic & 244 & 6.1 & 4.2 \\
\hline & Left lumbar & 206 & 5.2 & 4.4 \\
\hline & Right lumbar & 236 & 5.9 & 5.0 \\
\hline & Sacral & 207 & 5.2 & 5.2 \\
\hline & Subtotal & 1523 & 6.3 & 5.5 \\
\hline Total & & 2495 & 4.8 & 5.9 \\
\hline
\end{tabular}

Before the administration of drug on day 0 , the number of live motile mites (larvae, nymphs, and adults) was counted from 13 body regions of 40 hedgehogs using an otoscope (Piccolight ${ }^{\circledR}, \mathrm{KaWe}$, Berlin, Germany). Number of mites on approximately $0.5 \mathrm{~cm}^{2}$ of body surface was counted for each region.

\section{Discussion}

This study evaluated the efficacy and safety of $10 \%$ imidacloprid $+1 \%$ moxidectin (Advocate ${ }^{\circledR}$ for Cats, Bayer

Table 2 Determination of optimal dosage level of a combination of $10 \%$ imidacloprid and $1 \%$ moxidectin spot on against Caparinia tripilis in African pygmy hedgehogs (Atelerix albiventris)

\begin{tabular}{|c|c|c|c|c|c|c|c|c|c|}
\hline \multicolumn{2}{|c|}{ Days post treatment } & \multicolumn{4}{|c|}{0} & \multicolumn{4}{|c|}{3} \\
\hline Body regions & Dosage $(\mathrm{ml} / \mathrm{kg})^{a}$ & 0 & 0.1 & 0.4 & 1.6 & 0 & 0.1 & 0.4 & 1.6 \\
\hline \multirow[t]{3}{*}{ Ears } & Left & 11.6 & 8.4 & 15.5 & 15.8 & 7.0 & 0.0 & 0.0 & 0.0 \\
\hline & Right & 8.8 & 11.2 & 12.3 & 12.2 & 13.0 & 0.0 & 0.0 & 0.0 \\
\hline & Mean & 10.2 & 9.8 & 13.9 & 14.0 & 10.0 & 0.0 & 0.0 & 0.0 \\
\hline \multirow[t]{6}{*}{ Ventral } & Left pectoral & 1.8 & 2.4 & 2.2 & 1.6 & 3.4 & 0.0 & 0.0 & 0.0 \\
\hline & Right pectoral & 1.0 & 3.0 & 1.4 & 3.0 & 3.4 & 0.0 & 0.0 & 0.0 \\
\hline & Left abdominal & 0.8 & 1.0 & 1.8 & 1.6 & 2.2 & 0.0 & 0.0 & 0.0 \\
\hline & Right abdominal & 0.8 & 1.6 & 1.4 & 1.8 & 2.2 & 0.0 & 0.0 & 0.0 \\
\hline & Inguinal & 0.2 & 0.2 & 0.6 & 2.4 & 0.4 & 0.0 & 0.0 & 0.0 \\
\hline & Mean & 0.9 & 1.6 & 1.5 & 2.1 & 2.3 & 0.0 & 0.0 & 0.0 \\
\hline \multirow[t]{7}{*}{ Dorsal } & Dorsal neck & 10.2 & 3.6 & 12.2 & 5.0 & 6.2 & 0.2 & 0.0 & 0.0 \\
\hline & Left dorsal thoracic & 11.0 & 4.2 & 5.4 & 4.8 & 3.8 & 0.0 & 0.0 & 0.0 \\
\hline & Right dorsal thoracic & 7.4 & 5.0 & 4.6 & 4.0 & 3.4 & 0.0 & 0.0 & 0.0 \\
\hline & Left lumbar & 5.2 & 2.2 & 6.2 & 3.4 & 3.0 & 0.0 & 0.0 & 0.0 \\
\hline & Right lumbar & 4.0 & 2.2 & 8.0 & 5.4 & 3.0 & 0.0 & 0.0 & 0.0 \\
\hline & Sacral & 3.0 & 3.4 & 3.6 & 1.4 & 4.4 & 0.0 & 0.0 & 0.0 \\
\hline & Mean & 6.8 & 3.4 & 6.7 & 4.0 & 4.0 & 0.0 & 0.0 & 0.0 \\
\hline Mean & & 5.1 & 3.7 & 5.8 & 4.8 & 4.3 & 0.0 & 0.0 & 0.0 \\
\hline
\end{tabular}

${ }^{a}$ Advocate ${ }^{\circledR}$ for Cats (Bayer Animal Health $\mathrm{GmbH}$, Leverkusen, Germany) containing $10 \%$ imidacloprid $+1 \%$ moxidectin was administered once on the mid-dorsal part of each hedgehog. Each number represents mean number of live mites observed from 5 hedgehogs using an otoscope (Piccolight ${ }^{\circledR}$, KaWe, Berlin, Germany). Number of mites on approximately $0.5 \mathrm{~cm}^{2}$ of body surface was counted for each region. 
Table 3 Efficacy of a combination formulation of $10 \%$ imidacloprid $+1 \%$ moxidectin against naturally infested Caparinia tripilis in African pygmy hedgehogs (Atelerix albiventris)

\begin{tabular}{|c|c|c|c|c|c|c|c|c|c|c|c|}
\hline \multicolumn{2}{|c|}{ Groups } & \multicolumn{5}{|c|}{ Control } & \multicolumn{5}{|c|}{ Treatment } \\
\hline Body regions & Days post treatment ${ }^{a}$ & 0 & 3 & 9 & 16 & 30 & 0 & 3 & 9 & 16 & 30 \\
\hline \multirow[t]{3}{*}{ Ears } & Left & 5.2 & 5.9 & 10.7 & 15.7 & 7.9 & 4.7 & 0.0 & 0.0 & 0.0 & 0.0 \\
\hline & Right & 3.9 & 3.7 & 13.4 & 11.0 & 18.3 & 7.4 & 0.0 & 0.0 & 0.0 & 0.0 \\
\hline & Mean & 4.6 & 4.8 & 12.1 & 13.4 & 13.1 & 6.1 & 0.0 & 0.0 & 0.0 & 0.0 \\
\hline \multirow[t]{6}{*}{ Ventral } & Left pectoral & 2.8 & 0.6 & 1.8 & 0.8 & 0.5 & 1.8 & 0.0 & 0.0 & 0.0 & 0.0 \\
\hline & Right pectoral & 1.3 & 0.5 & 1.6 & 0.6 & 1.2 & 1.8 & 0.0 & 0.0 & 0.0 & 0.0 \\
\hline & Left abdominal & 0.8 & 0.6 & 1.4 & 1.0 & 1.1 & 1.3 & 0.0 & 0.0 & 0.0 & 0.0 \\
\hline & Right abdominal & 0.9 & 0.6 & 1.0 & 0.5 & 1.0 & 2.9 & 0.0 & 0.0 & 0.0 & 0.0 \\
\hline & Inguinal & 0.5 & 0.0 & 0.8 & 0.9 & 1.3 & 1.5 & 0.0 & 0.0 & 0.0 & 0.0 \\
\hline & Mean & 1.3 & 0.5 & 1.3 & 0.8 & 1.0 & 1.9 & 0.0 & 0.0 & 0.0 & 0.0 \\
\hline \multirow[t]{7}{*}{ Dorsal } & Dorsal neck & 9.1 & 13.8 & 8.3 & 16.0 & 7.4 & 8.1 & 0.0 & 0.0 & 0.0 & 0.0 \\
\hline & Left dorsal thoracic & 5.7 & 15.3 & 7.3 & 9.6 & 5.6 & 11.9 & 0.0 & 0.0 & 0.0 & 0.0 \\
\hline & Right dorsal thoracic & 6.4 & 12.2 & 7.6 & 9.0 & 5.8 & 7.5 & 0.0 & 0.0 & 0.0 & 0.0 \\
\hline & Left lumbar & 6.5 & 9.6 & 8.2 & 7.0 & 5.5 & 5.6 & 0.0 & 0.0 & 0.0 & 0.0 \\
\hline & Right lumbar & 7.2 & 10.8 & 8.2 & 6.9 & 5.1 & 6.6 & 0.0 & 0.0 & 0.0 & 0.0 \\
\hline & Sacral & 10.5 & 11.4 & 8.9 & 10.7 & 9.0 & 4.5 & 0.0 & 0.0 & 0.0 & 0.0 \\
\hline & Mean & 7.6 & 12.2 & 8.1 & 9.9 & 6.4 & 7.4 & 0.0 & 0.0 & 0.0 & 0.0 \\
\hline Mean & & 4.7 & 6.5 & 6.1 & 6.9 & 5.4 & 5.0 & 0.0 & 0.0 & 0.0 & 0.0 \\
\hline \% Efficacy & & & & & & & & 100.0 & 100.0 & 100.0 & 100. \\
\hline
\end{tabular}

${ }^{a}$ Advocate ${ }^{\circledR}$ for Cats (Bayer Animal Health $\mathrm{GmbH}$, Leverkusen, Germany) containing $10 \%$ imidacloprid + $1 \%$ moxidectin was administered once on the mid-dorsal part of each hedgehog. For abbreviation, refer to Table 1. Each number represents mean number of live mites observed from 10 hedgehogs using an otoscope (Piccolight ${ }^{\circledR}$, KaWe, Berlin, Germany). Number of mites on approximately $0.5 \mathrm{~cm}^{2}$ of body surface was counted for each region. The percentage of efficacy $(\%)=$ $(y-x) / y^{*} 100(x=$ number of hedgehogs observed with live mites in the test group for each observation date, $y=$ total number of hedgehogs in the test group for each observation date).

Animal Health GmbH, Leverkusen, Germany) against naturally infested Caparinia tripilis in African pygmy hedgehogs. Though the drug we tested was primarily formulated for cat use, our results showed excellent efficacy and safety against the mange mite infestation in hedgehogs, too. The dosage level of $0.1 \mathrm{ml} / \mathrm{Kg}$ that was determined in Experiment 1 was also the recommended dosage for cats. Even on the third day post treatment, no hedgehogs in the treated group showed live mites in any body regions, which continued until the end of the experiment (30 days). In our study, side effects such as ataxia, depression, nausea, and weight fluctuation were not observed during the whole period of study. With the ease of use of a spot-on medication, especially for pet owners, the drug appears to be an ideal solution for the control of mange mite infestation in exotic pets such as hedgehogs.

Moxidectin, a macrocyclic lactone disaccharide, is about 100 times more lipophilic than ivermectin [10] and remains in the plasma much longer than ivermectin [19]. Once the drug enters the blood stream, it is stored in fat and slowly released, metabolized and excreted mainly via the feces but also appears on the skin where it kills surface-living mites. The miticidal efficacy of Advocate $^{\circledR}$ for Cats (10\% imidacloprid $+1 \%$ moxidectin) against $C$. tripilis in this study may therefore be due to the activity of moxidectin because even those mites remotely located in the external ear canal were successfully killed. It has been reported that notoedric mange caused by Notoedres cati in a hedgehog was also treated with $0.3 \mathrm{mg} / \mathrm{Kg}$ moxidectin (Cydectin ${ }^{\circledR} 1 \%$ injectable, Fort Dodge, IA, USA), administered subcutaneously, which was repeated after 10 days. Clinical signs improved significantly 10 days after the initial treatment [20]. Though the report did not record the number of mites before and after the treatment, moxidectin seemed to be effective for external parasite infestations in hedgehogs.

It appears that hedgehogs became popular as pets in the United States in the early 1990s when "Hazel the Hedgehog" turned up in an American comic strip in 1993 and "Sonic the Hedgehog" began to appear in the cartoon that bore his name [21]. The North American Hedgehog Association estimated that there were approximately 2,000 quality breeding animals in the United States in 1995 which remained rather stable in 2000 [21]. Although hedgehogs are legal to keep as pets in all of Canada and most areas of the United States of America, it is illegal to own them as pets in some US states and some Canadian municipalities, probably due to the 

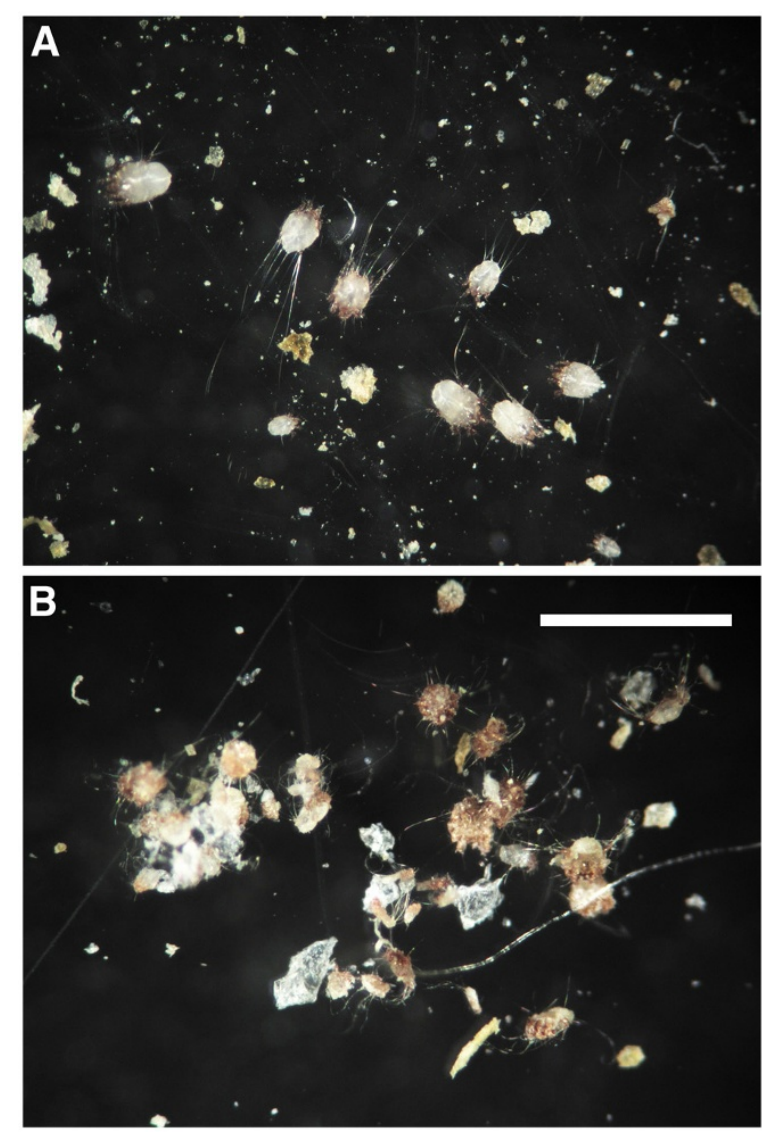

Figure 4 Various stages of both live and dead mites of Caparinia tripilis collected from hedgehogs (Atelerix albiventris) A, Live mites collected from hedgehogs in the control group without any chemotherapy by skin scraping in the posterior dorsal neck region. Live mites can be easily distinguished from dead mites by their active motility and retention of their body conformation. B, Dead mites collected from hedgehogs 3 days after treatment with $0.1 \mathrm{ml} / \mathrm{kg}$ b.w. of $10 \%$ imidacloprid $+1 \%$ moxidectin (Advocate ${ }^{\circledR}$ for Cats, Bayer Animal Health GmbH, Leverkusen, Germany). Bar $=1$ $\mathrm{mm}$.

ability of some hedgehog species to carry foot and mouth disease [22]. Only the African pygmy hedgehog can be legally kept in most European countries [21].

Caparinia infestion of hedgehog was first reported in England in 1889 by Michael who identified the mite as Symbiotes (=Caparinia) tripilis. The mite was then found in hedgehogs from Germany and New Zealand [2,23], and it has also been introduced to New Mexico, United States, through breeding colonies of African hedgehogs for sale as pets [7]. In Asia, an outbreak of C. tripilis infestation in a colony of African pygmy hedgehogs from Korea was reported in 2012 [24]. Human ringworm and salmonellosis cases acquired from African pygmy hedgehogs have also been reported [25,26], but Caparinia tripilis does not appear to be zoonotic [27].

\section{Conclusions}

Although there has been a great increase in the population of hedgehogs as pets, limited studies on the control of ectoparasitic diseases of hedgehogs are available. This report suggests that a combination formulation of 0.1 $\mathrm{ml} / \mathrm{Kg}$ of $10 \%$ imidacloprid $+1 \%$ moxidectin spot-on for cats is also useful for the control of Caparinia tripilis in hedgehogs.

\section{Competing interests}

The authors declare that the conceptual design, the content or any other scientific aspect have not been influenced.

\section{Authors' contributions}

KRK and SSS planned the study design. KRK and KSA performed laboratory work. DSO analyzed the data. KRK drafted the manuscript. SSS provided substantial improvement of the manuscript and scientific supervision of the study. All authors approved the final version of the manuscript.

\section{Acknowledgements}

This work was supported in part by the Ministry of Education and Human Resources Development through the Brain Korea 21 Project in Korea. Publication fee for this study was paid by Bayer Animal Health $\mathrm{GmbH}$.

\section{Author details}

${ }^{1}$ Department of Parasitology, College of Veterinary Medicine, Chonnam National University, Gwangju 500-757, Korea. ${ }^{2}$ Biotherapy Human Resources Center, College of Veterinary Medicine, Chonnam National University, Gwangju 500-757, Korea.

Received: 27 February 2012 Accepted: 28 July 2012 Published: 7 August 2012

\section{References}

1. Michael AD: On some unrecorded parasitic Acari found in Great Britain. Zool J Linn Soc 1889, 20:400-406.

2. Brockie RE: The hedgehog mange mite, Caparinia tripilis, in New Zealand. N Z Vet J 1974, 22:243-247.

3. Sweatman GK: Parasitic mites of non-domesticated animals in New Zealand. N Z Entomol 1962, 3:15-23.

4. Gerson L, Boever W: Acariasis (Caparinia sp.) in hedgehogs (Erinaceusspp.): diagnosis and treatment. J Zoo Anim Med 1983, 14:17-19.

5. Letcher JD: Amitraz as a treatment for acariasis in African hedgehogs (Atelerix albiventris). J Zoo Anim Med 1988, 19:24-29.

6. Holstege CP, Baer AB: Insecticides. Curr Treat Options Neurol 2004, 6:17-23.

7. Staley E, Staley E, Behr M: Use of permethrin as a miticide in the African hedgehog (Atelerix albiventris). Vet Hum Toxicol 1994, 36:138.

8. Cobb R, Boeckh A: Moxidectin: a review of chemistry, pharmacokinetics and use in horses. Parasit Vectors 2009, 2(Suppl 2):S5.

9. Zulalian J, Stout SJ, da Cunha AR, Garces T, Miller P: Absorption, tissue distribution, metabolism, and excretion of moxidectin in cattle. J Agric Food Chem 1994, 42:381-387.

10. Hayes PW: Moxidectin: understanding the unique persistent anthelmintic activity of this second generation macrocyclic lactone. In Proc Aust Vet Assoc; March 6-11th. Canberra, Australia: Australian Veterinary Association; 1994.

11. Heine J, Krieger K, Dumont P, Hellmann K: Evaluation of the efficacy and safety of imidacloprid $10 \%$ plus moxidectin $2.5 \%$ spot-on in the treatment of generalized demodicosis in dogs: results of a European field study. Parasitol Res 2005, 97(Suppl 1):S89-96.

12. Farkas R, Germann T, Szeidemann Z: Assessment of the ear mite (Otodectes cynotis) infestation and the an imidacloprid plus moxidectin combination in the treatment of otoacariosis in a Hungarian cat shelter. Parasitol Res 2007, 101:S35-S44.

13. Dryden MW, Payne PA, Smith V, Hostetler J: Efficacy of imidacloprid + moxidectin and selamectin topical solutions against the KS1Ctenocephalides felis flea strain infesting cats. Parasit Vectors 2011, 4:174. 
14. Le Sueur C, Bour S, Schaper R: Efficacy and safety of the combination imidacloprid $10 \%$ / moxidectin $1.0 \%$ spot-on $\left(\right.$ Advocate $^{(R)}$ ) spot-on for small cats and ferrets) in the treatment of ear mite infection (Otodectes cynotis) in ferrets. Parasitol Res 2011, 109(Suppl 1):S149-156.

15. Downs WG: Polyvinyl alcohol: a medium for mounting and clearing biological specimens. Science 1943, 97:539.

16. Lawrence R: A new mange-mite from the Cape polecat. Ann Trop Med Parasitol 1955, 49:54.

17. Sweatman GK: Life history, non-specificity, and revision of the genus Chorioptes, a parasitic mite of herbivores. Can J Zool 1957, 35:641-689.

18. Sweatman GK: Biology of Otodectes cynotis, the ear canker mite of carnivores. Can J Zool 1958, 36:849-862.

19. Craven J, Bjorn H, Hennessy D, Friis C, Nansen P: Pharmacokinetics of moxidectin and ivermectin following intravenous injection in pigs with different body compositions. J Vet Pharmacol Ther 2001, 24:99-104.

20. Pantchev N, Hofmann T: Notoedric mange caused by Notoedres catiin a pet African pygmy hedgehog (Atelerix albiventris). Vet Rec 2006, 158:59.

21. Vriends MM: Hedgehogs - A complete pet owner's manual. 2nd edition. Hauppauge, NY: Barron's Educational Series; 2000.

22. McLauchlan JD, Henderson WM: The occurrence of foot-and-mouth disease in the hedgehog under natural conditions. J Hyg (Lond) 1947, 45:474-479.

23. Stocker L: The complete hedgehog. London: Chatto \& Windus Ltd; 1987.

24. Kim DH, Oh DS, Ahn KS, Shin SS: An Outbreak of Caparinia tripilisin a Colony of African Pygmy Hedgehogs (Atelerix albiventris) from Korea. Korean J Parasitol 2012, 50:151-156.

25. CDC: African pygmy hedgehog-associated salmonellosis-Washington, 1994. Morb Mortal Wkly Rep 1995, 44:462-463.

26. Philpot CM, Bowen RG: Hazards from hedgehogs: two case reports with a survey of the epidemiology of hedgehog ringworm. Clin Exp Dermatol 1992, 17:156-158.

27. Chitty J, Hendricks A: Zoonotic skin disease in small animals. In Pract 2007, 29:1292-97.

doi:10.1186/1756-3305-5-158

Cite this article as: Kim et al:: Efficacy of a combination of $10 \%$

imidacloprid and $1 \%$ moxidectin against Caparinia tripilis in African pygmy hedgehog (Atelerix albiventris). Parasites \& Vectors 2012 5:158.

\section{Submit your next manuscript to BioMed Central and take full advantage of:}

- Convenient online submission

- Thorough peer review

- No space constraints or color figure charges

- Immediate publication on acceptance

- Inclusion in PubMed, CAS, Scopus and Google Scholar

- Research which is freely available for redistribution 